\title{
Tratamento cirúrgico da fenda do lóbulo de orelha surgery
}

\author{
Lucas Gomes Patrocínio', Rodrigo Márcio \\ Morais ${ }^{2}$,José Edmundo Pereira ${ }^{3}$,José Antônio \\ Patrocínio $^{4}$
}

Palavras-chave: deformidades da orelha, estética, procedimentos cirúrgicos reconstrutivos.

Keywords: ear deformities, esthetics, reconstructive surgical procedures.

\section{Resumo / Summary}

$\mathrm{O}$ lóbulo de orelha ocupa posição única entre as estruturas da face e tem relevante importância devido ao uso de adornos e jóias. Novos hábitos têm motivado uma maior procura dos pacientes para tratamento especializado em alterações estéticas do lóbulo de orelha. Objetivo: Apresentar e discutir a técnica utilizada nos Serviços de Otorrinolaringologia da Faculdade de Medicina da Universidade Federal de Uberlândia (FAMED-UFU) e do Hospital Santa Genoveva, no tratamento da fenda do lóbulo de orelha. Pacientes e Métodos: Foram avaliados 25 pacientes, de janeiro de 2003 a maio de 2005, apresentando fenda no lóbulo, totalizando 35 orelhas. Em todos foi utilizada a técnica que denominamos "Cirurgia do Brinco". Resultados: Dos 35 casos, 32 apresentaram resultados satisfatórios, 1 apresentou déficit estético notado somente pelo cirurgião e 2 apresentaram déficit estético notado pelo paciente e pelo cirurgião, necessitando cirurgia revisional. Nestes, houve desnível na margem livre inferior. O outro caso com déficit foi funcional devido ao fechamento do orifício do lóbulo. Conclusões: Consideramos a técnica da "Cirurgia do Brinco" uma técnica inovadora, de fácil realização e com bons resultados estéticos e funcionais sendo, portanto, para os autores, a técnica de eleição na correção da fenda do lóbulo de orelha.
$T_{1}$ structures and has its own importance when we consider the secular tradition of wearing decorations and jewels on it. Aim: To present and discuss the technique used in the Departments of Otolaryngology of the Federal University of Uberlândia (FAMED-UFU) and the Hospital Santa Genoveva, in the treatment of earlobe clefts. Patient and Methods: Twenty-five patients (35 ears) with earlobe clefts were evaluated, from January 2003 to May 2005. In all these cases we used the technique we call "Surgery of the Ear Ring ". Results: Of the 35 cases, 32 presented satisfactory results, 1 presented with an aesthetic deficit only noticed by the surgeon, and 2 presented aesthetic deficits noticed by both the patient and surgeon, needing a "second look" surgery. In these, there was a notch in the lower free border. The other case with deficit was a functional one caused by the closing of the ear lobe hole. Conclusions: We consider this technique an innovative one, of easy accomplishment, and with good aesthetic and functional results. Therefore, it is the authors preferred technique for the correction of earlobe clefts.

\footnotetext{
${ }^{1}$ Médico, Residente do Serviço de Otorrinolaringologia da Faculdade de Medicina da Universidade Federal de Uberlândia.

${ }^{2}$ Médico, Residente do Serviço de Otorrinolaringologia da Faculdade de Medicina da Universidade Federal de Uberlândia.

${ }^{3}$ Cirurgião Dermatológico, Chefe da Divisão de Cirurgia Dermatológica do Serviço de Otorrinolaringologia da Faculdade de Medicina da Universidade Federal de Uberlândia.

${ }^{4}$ Professor Titular, Chefe do Serviço de Otorrinolaringologia da Faculdade de Medicina da Universidade Federal de Uberlândia. Serviço de Otorrinolaringologia do Hospital Santa Genoveva e do Hospital de Clínicas da Universidade Federal de Uberlândia, Uberlândia, Minas Gerais, Brasil. Endereço para correspondência: Lucas Gomes Patrocínio - Rua XV de Novembro 327 apto. 1600 Centro Uberlândia MG 38400-214. Tel/Fax: (0xx34) 3215-1143 - E-mail: lucaspatrocinio@triang.com.br

Este artigo foi submetido no SGP (Sistema de Gestão de Publicações) da RBORL em 6 de dezembro de 2005.

Artigo aceito em 2 de maio de 2006
} 


\section{INTRODUÇÃO}

O lóbulo de orelha ocupa posição única entre as estruturas da face e têm relevante importância devido à tradição secular do uso de adornos e jóias neste local. A prática de perfuração do lóbulo da orelha data de tempos antigos, e, dependendo da cultura, pode ter ocorrido até como uma obrigação social. As sociedades latino-americanas têm usado rotineiramente brincos nos recém-nascidos do sexo feminino para diferenciar dos do sexo masculino. Nas tribos de Ivan e Kayan, na África, os lóbulos eram adornados com grandes e pesados brincos, causando aumento e alongamento do orifício do lóbulo ${ }^{1}$.

Além disso, observa-se uma tendência atual de utilizar um maior número de adornos nesta região, provocando maior tensão local e alterações estruturais decorrentes desses novos hábitos, mesmo no sexo masculino. Tudo isso tem motivado uma maior procura dos pacientes para tratamento especializado em alterações estéticas do lóbulo de orelha².

Existem várias causas e/ou alterações que requerem tratamento cirúrgico. Entre elas podemos citar ${ }^{3,4}$ :

- Fendas ou lacerações de lóbulo de orelha secundárias a trauma;

- Alterações congênitas;

- Envelhecimento facial;

- Quelóides;

- Tumores de lóbulo de orelha.

Numerosas técnicas têm sido descritas para correção da fenda do lóbulo de orelha ${ }^{1,5,6,7}$ :

- Sutura direta;

- Zetaplastias;

- Ritidoplastia com correção do lóbulo;

- Retalhos em V;

- Retalhos em L;

- Outras técnicas que podem ser as citadas acima com algumas variantes ou até mesmo uma combinação entre elas.

O objetivo deste trabalho é apresentar e discutir a técnica utilizada no Serviços de Otorrinolaringologia da Faculdade de Medicina da Universidade Federal de Uberlândia (FAMED-UFU) e do Hospital Santa Genoveva, no tratamento da fenda do lóbulo de orelha.

\section{PACIENTES E MÉTODOS}

\section{Pacientes}

Foram avaliados 25 pacientes, no período de janeiro/03 a maio/05 apresentando fenda no lóbulo de orelha, totalizando 35 orelhas. Todos os pacientes eram do sexo feminino, com idade variando de 16 a 58 anos (média de 24,5 anos). Com a relação à cor da pele, 10 eram da cor branca, 12, parda e 3, negra.

Os pacientes foram classificados em dois grupos:

- Fenda completa: normalmente unilateral resultante de trauma direto local, como, por exemplo, uma tração súbita do brinco provocando transsecção total;

- Fenda incompleta: normalmente bilateral e mais freqüente na mulher idosa com hábito de usar brincos pesados durante vários anos. Esses pacientes foram subdivididos em 3 grupos:

1. Tipo I: a extensão da fenda não ultrapassa metade da distância entre orifício inicial e borda inferior do lóbulo da orelha;

2. Tipo II: a extensão da fenda ultrapassa a metade da distância entre orifício inicial e borda inferior do lóbulo da orelha;

3. Tipo III: crescimento progressivo da fenda até se tornar completa.

O presente trabalho foi aprovado pelo Comitê de Ética sobre parecer no 015/05.

\section{Técnica Cirúrgica}

Em todos os 35 casos foi utilizada a técnica descrita abaixo que denominamos "Cirurgia do Brinco".

1. Assepsia e antissepsia com povidine tópico;

2. Anestesia local com lidocaína $2 \%$ sem vasoconstritor;

3. Incisão longitudinal completando a fenda do lóbulo em direção à borda inferior, dividindo o lóbulo em 2 metades (Figuras 1 e 2);

4. Escarificação de um dos retalhos do lóbulo criando uma área cruenta (Figuras 3 e 4);

5. Incisão longitudinal no outro retalho do lóbulo, com espessura de $2,0 \mathrm{~mm}$ e criando um retalho de 5,0 x 2,0 mm (mantendo uma das faces epitelizada) (Figura 5);

6. Excisão transversal de $2,0 \mathrm{~mm}$ da borda inferior do retalho (Figura 6);

7. Rotação do retalho de tal forma que as bordas epitelizadas constituam o orifício do lóbulo e sutura com mononylon 5-0 (Figura 7);

8. Sutura dos retalhos do lóbulo iniciando pela margem livre inferior com mononylon 5-0 (Figura 8, 9 e 10);

9. Curativo com micropore.

10. Os pacientes receberam alta em uso de cefalexina e dipirona. Retornaram com 10 a 15 dias para retirada dos pontos e no $30^{\circ}$ e $60^{\circ}$ pós-operatório para reavaliação. $\mathrm{Na}$ última consulta, a cirurgia foi avaliada subjetivamente tanto pelo médico quanto pelo paciente. 


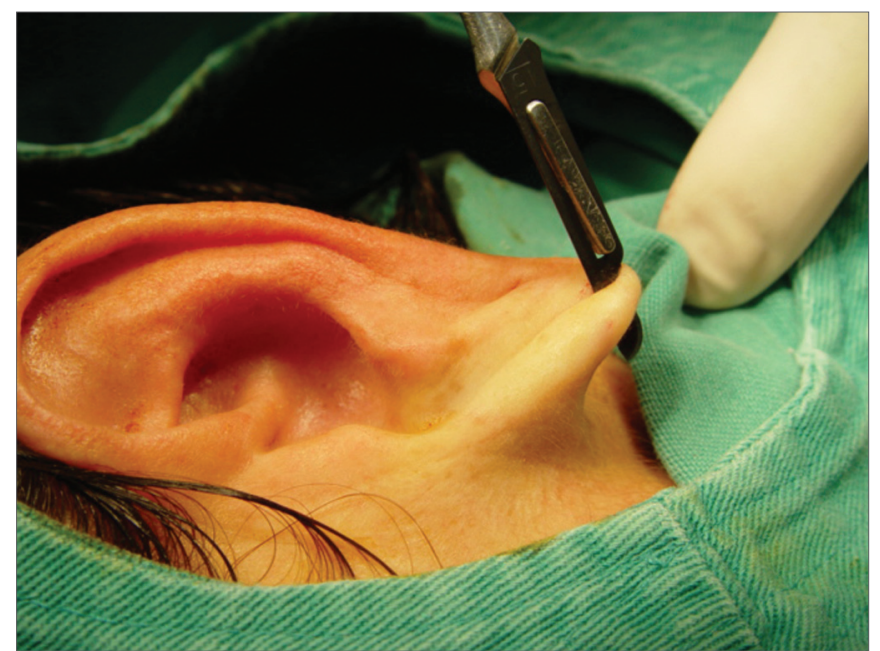

Figura 1. Fotografia demonstrando incisão longitudinal completando a fenda do lóbulo em direção à borda inferior, dividindo o lóbulo em 2 metades.

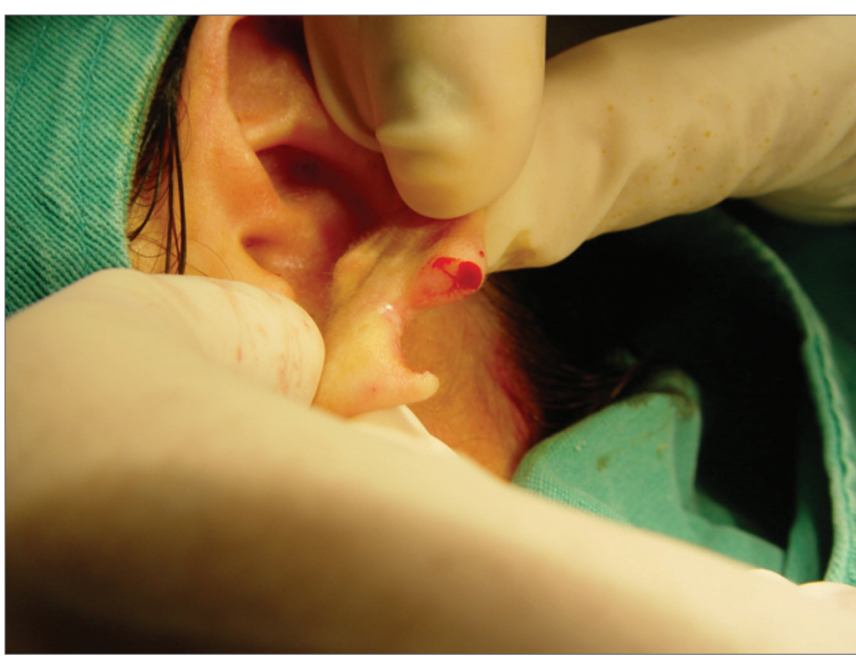

Figura 2. Fotografia demonstrando o lóbulo dividido em 2 retalhos.

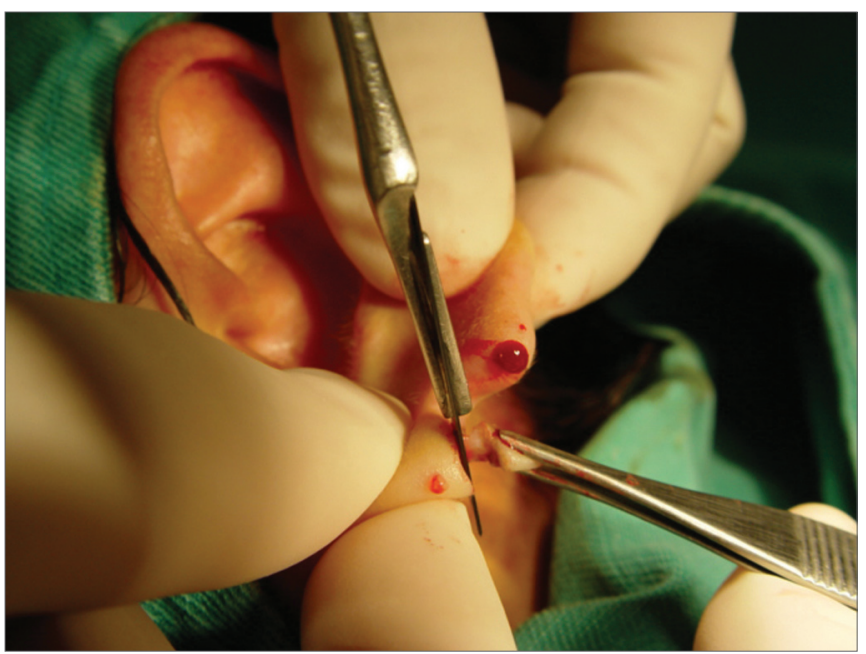

Figura 3. Fotografia demonstrando escarificação do retalho anterior do lóbulo.

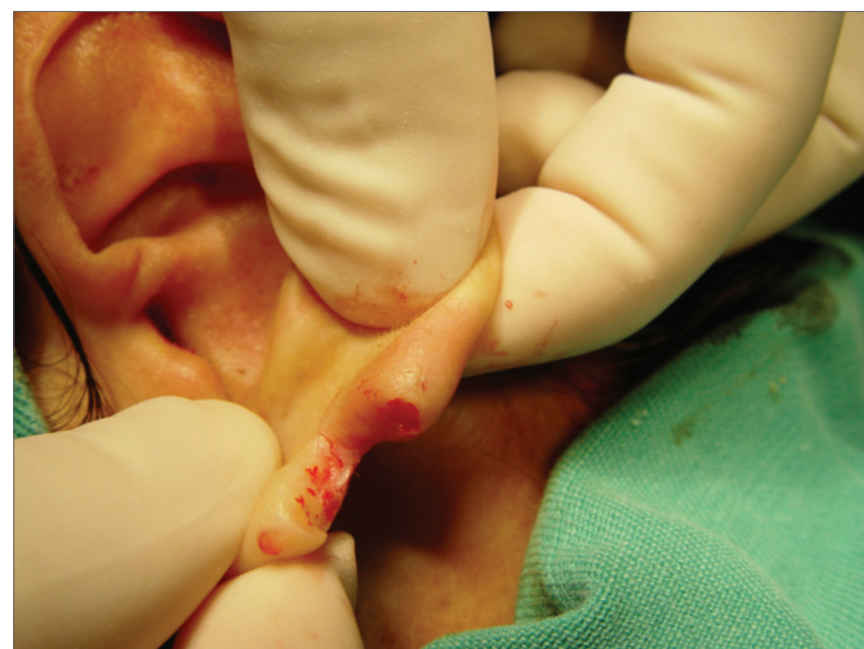

Figura 4. Fotografia demonstrando retalho anterior do lóbulo apresentando-se com face posterior totalmente cruenta.

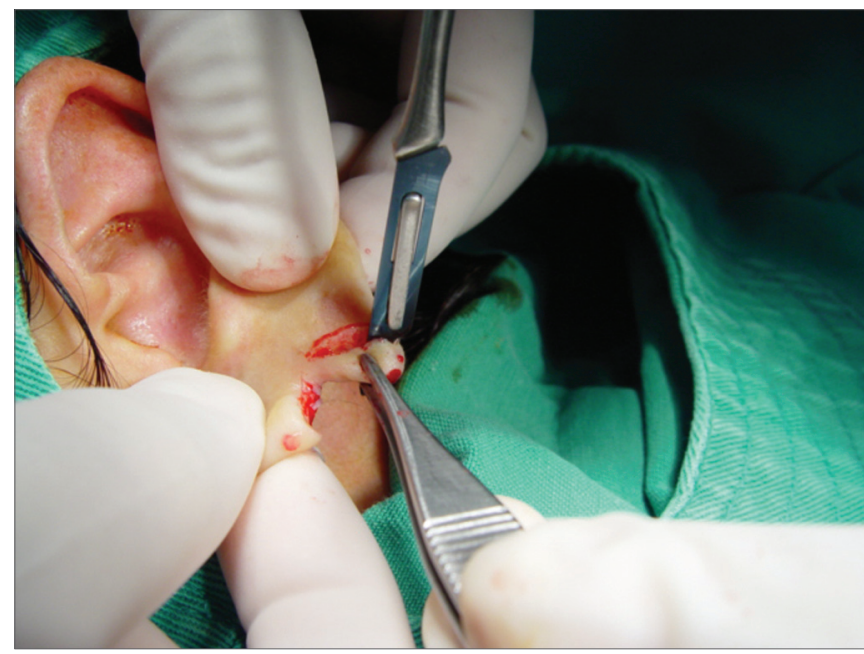

Figura 5. Fotografia demonstrando incisão longitudinal no retalho posterior do lóbulo, com espessura de $2,0 \mathrm{~mm}$, criando um novo retalho de $5,0 \times 2,0 \mathrm{~mm}$.

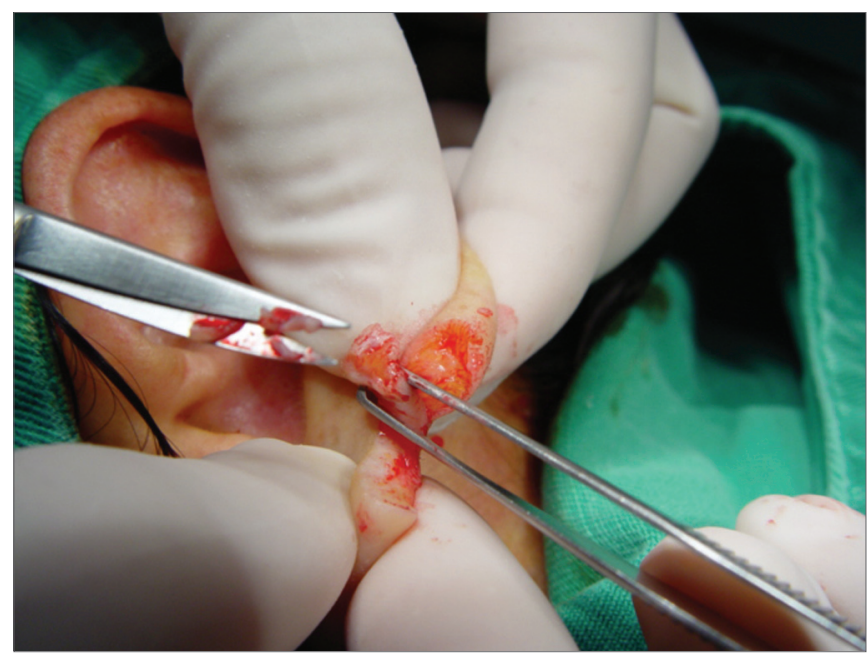

Figura 6. Fotografia demonstrando excisão transversal de 2,0 mm da borda inferior do retalho central. 


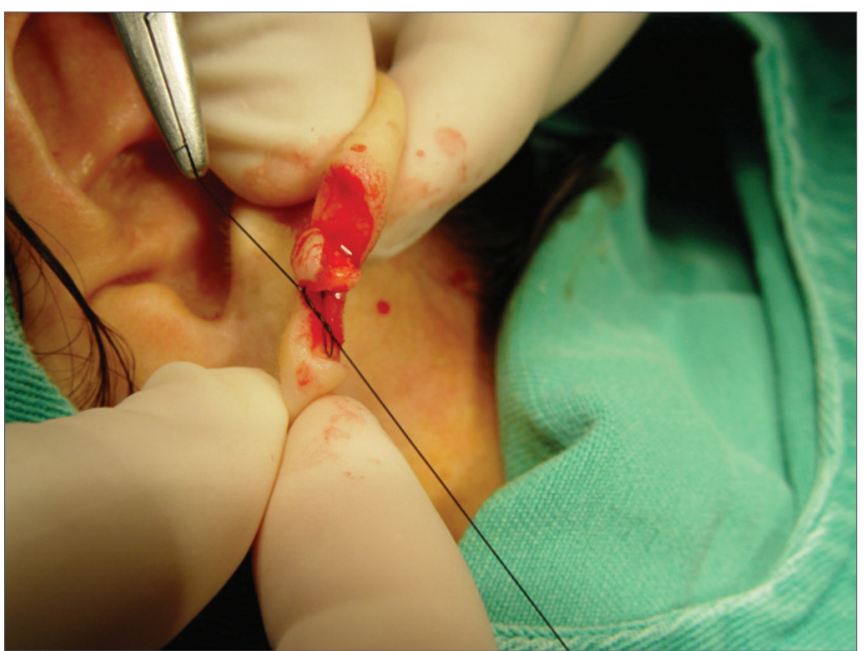

Figura 7. Fotografia demonstrando rotação do retalho central de tal forma que as bordas epitelizadas constituam o orifício do lóbulo e sutura com mononylon 5-0.

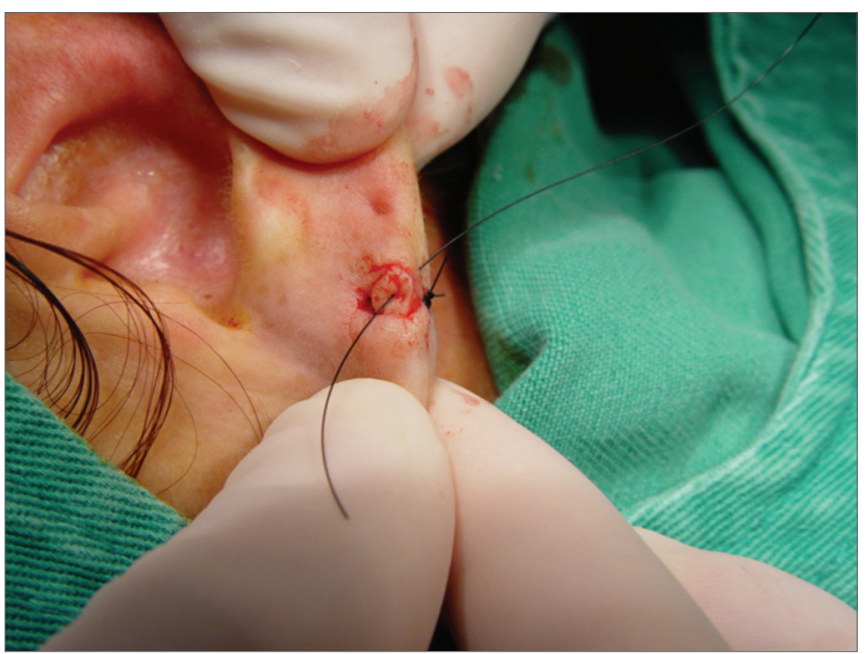

Figura 8. Fotografia demonstrando sutura dos retalhos do lóbulo iniciando pela margem livre inferior com mononylon 5-0.

\section{RESULTADOS}

Das 35 orelhas submetidas a "Cirurgia do Brinco", 22 apresentavam fenda completa e 13 incompleta, sendo destas $3 \mathrm{com}$ fenda tipo I e $10 \mathrm{com}$ fenda tipo II.

A avaliação subjetiva final da cirurgia depende dos pontos de vista do paciente e do cirurgião, que, em alguns casos, diferem. Dos 35 casos, 32 apresentaram resultados satisfatórios, 1 apresentou déficit estético notado somente pelo cirurgião e 2 apresentaram déficit estético notado pelo paciente e pelo cirurgião, necessitando cirurgia revisional (Quadro 1).

A cirurgia revisional foi necessária nesses dois casos em que houve desnivel na margem livre inferior. Tal complicação deve ser evitada com técnicas simples de sutura, fazendo com que a entrada do fio seja mais próxima da

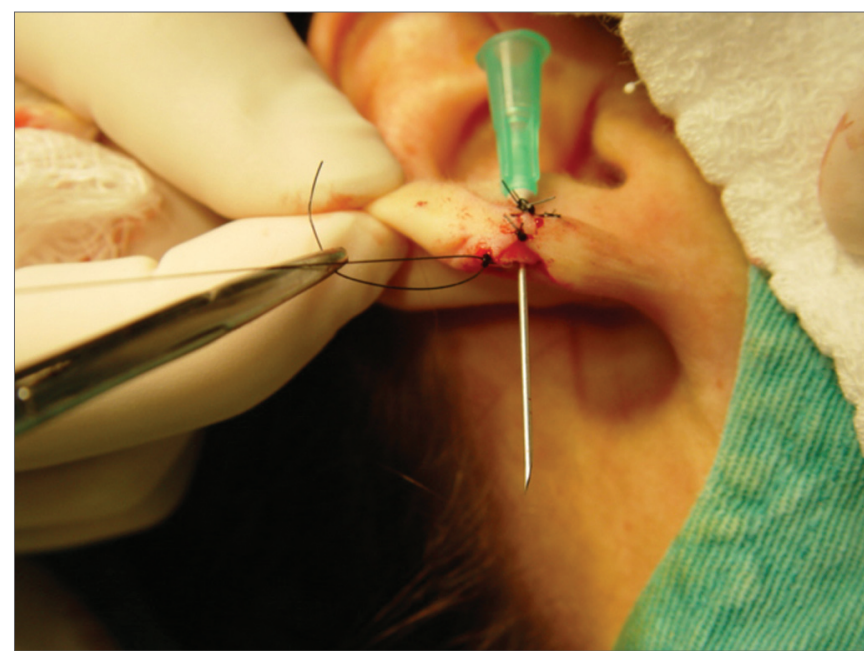

Figura 9. Fotografia demonstrando sutura dos retalhos do lóbulo com nylon 5-0 e presença da agulha no orifício final para colocação do brinco.

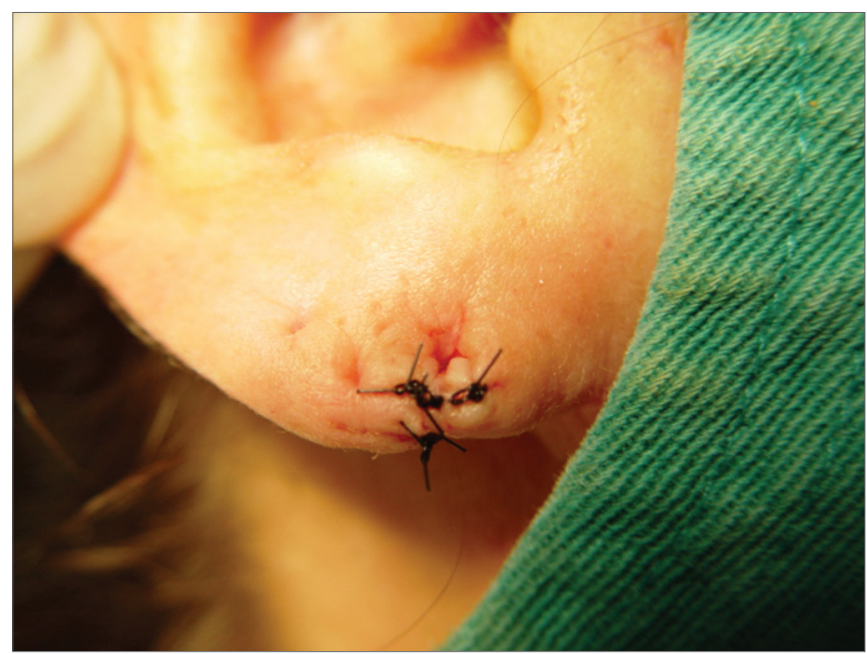

Figura 10. Fotografia demonstrando aspecto final da cirurgia. Nota-se a presença do orifício para a colocação do brinco todo epitelizado.

Quadro 1. Distribuição dos pacientes submetidos a tratamento cirúrgico do lóbulo da orelha que apresentaram déficit funcional e/ou estético.

\begin{tabular}{|c|c|c|c|c|c|c|}
\hline $\mathrm{N}$ & Sexo & Idade & $\begin{array}{l}\text { Cor da } \\
\text { Pele }\end{array}$ & $\begin{array}{l}\text { Tipo de } \\
\text { Fenda }\end{array}$ & Falha & Tratamento \\
\hline 3 & $\mathrm{~F}$ & 18 & Branca & Completa & $\begin{array}{c}\text { Entalhe } \\
\text { borda }\end{array}$ & $\begin{array}{l}\text { Revisão } \\
\text { margem }\end{array}$ \\
\hline 12 & $\mathrm{~F}$ & 27 & Parda & Incompleta II & $\begin{array}{c}\text { Entalhe } \\
\text { borda }\end{array}$ & $\begin{array}{l}\text { Revisão } \\
\text { margem }\end{array}$ \\
\hline 27 & $\mathrm{~F}$ & 24 & Branca & Completa & $\begin{array}{l}\text { Sem } \\
\text { orifício }\end{array}$ & $\begin{array}{l}\text { Novo ori- } \\
\text { fício }\end{array}$ \\
\hline
\end{tabular}


margem do lado mais alto e mais longe da margem do lado mais baixo.

O outro caso com déficit foi funcional devido ao fechamento do orifício do lóbulo, porém o paciente não notou. Nessa situação, novo orifício pode ser realizado da forma tradicional após 30 dias de cirurgia.

Não houve complicações como formação de quelóides, hipo ou hiperpigmentação, granuloma na incisão, deiscência de sutura e necrose de pele.

\section{DISCUSSÃO}

Existem muitas técnicas descritas para o tratamento cirúrgico do lóbulo da orelha. Em 1954, McLaren sugeriu pequena escarificação das bordas da fenda e sutura simples das margens ${ }^{2,5}$. Além de não manter o orifício do lóbulo, a sutura simples favorece a formação do entalhe.

Boo-chai, em 1961, propôs a excisão de parte das bordas da fenda e sutura abaixo do orifício original. Pardue, em 1973, propôs a ressecção das bordas da fenda, deixando um pedaço de pele na porção superior de um dos lados que será utilizado para constituir o orifício do lóbulo ${ }^{3,5}$. Estas duas técnicas, apesar de tentarem manter o orifício, favorecem a formação do entalhe ou desnível na margem inferior do lóbulo.

Hamilton e La Rossa, em 1975, descreveram uma técnica semelhante à de Pardue, associada a uma zetaplastia na tentativa de minimizar a formação do entalhe. Embora esta técnica tentasse manter o orifício e evitar o entalhe, é tecnicamente mais difícil que a técnica proposta neste trabalho. Argamasso, em 1978, descreveu uma técnica semelhante que deixava pele intacta próximo ao orifício original, e em cada metade do lóbulo criava 2 retalhos triangulares para, em seguida, suturá-los 3,5 .

Harak, em 1982, propôs a excisão de tecido na superfície anterior de uma das bordas. Em seguida, retira-se a mesma quantidade de tecido na superfície posterior da outra borda. Esta técnica também não preserva o orifício do lóbulos.

Kalimuthu et al. propuseram a técnica do retalho em "V". Fatah (1985) e Fearon \& Cuadros (1990), a técnica do retalho em "L" que novamente não mantém o orifício do lóbulo². Pode-se observar, então, que nenhuma das técnicas existentes consegue, ao mesmo tempo, resultados satisfatórios como os obtidos na "Cirurgia do Brinco". Esta técnica permite um bom resultado estético, sem entalhe ou desnível na margem livre, e ao mesmo tempo, mantém um orifício no lóbulo com boa resistência, além de ser tecnicamente de fácil realização.

Os resultados insatisfatórios foram considerados dentro da normalidade esperada e de baixa complexidade, isto é, foram resolvidos facilmente e os pacientes ficaram satisfeitos com o resultado final. Não houve relação do insucesso com tipo de pele, idade, sexo ou tipo de fenda.

\section{CONCLUSÃO}

Consideramos a técnica da "Cirurgia do Brinco" uma técnica inovadora, de fácil realização e com bons resultados estéticos e funcionais sendo, portanto, na opinião dos autores, a técnica de eleição na correção da fenda do lóbulo de orelha.

\section{REFERÊNCIAS BIBLIOGRÁFICAS}

1. Abrahams PH. Earlobe Z-plasty in Borneo. Plast Reconstr Surg 1974;53:548-50.

2. Fearon J, Cuadros CL. Cleft earlobe repair. Ann Plast Surg 1990;24(3):252-7.

3. Venkatramani H. A new technique in closure of wide clefts of earlobule. Plast Reconstr Surg 1999;104(1):296-7.

4. Clevens RA, Baker RS. Plastic and reconstructive surgery of the earlobe. Facial Plast Surg 1995;11(4):301-9.

5. Rich JD, Gottlieb V, Shesol BF. A simple method for correction of the pixie earlobe. Plast Reconstr Surg 1982;69(1):136-8

6. Effendi SH. Reconstruction of the middle-aged torn earlobe: a new method. Br J Plast Surg 1988;41(2):174-6.

7. Blanco-Davila F, Vasconez HC. The cleft earlobe: a review of methods of treatment. Ann Plast Surg 1994;33(6):677-80. 